\title{
Fungos micorrízicos arbusculares no crescimento e nutrição de mudas de jenipapeiro ${ }^{1}$
}

\author{
Arbuscular mycorrhizal fungi in the growth and nutrition of jenipapo fruit tree \\ seedlings
}

\author{
Ana Cristina Fermino Soares ${ }^{2}$, Carla da Silva Sousa ${ }^{2 *}$, Marlon da Silva Garrido ${ }^{3}$ e Francisco de Sousa Lima ${ }^{4}$
}

\begin{abstract}
Resumo - Alguns trabalhos têm demonstrado que a inoculação de fungos micorrízicos arbusculares (FMA) na produção de mudas apresenta grande potencial para o desenvolvimento de um cultivo racional e eficiente de mudas de fruteiras. O objetivo neste trabalho foi avaliar a inoculação de fungos micorrízicos arbusculares no crescimento e nutrição de mudas de jenipapeiro (Genipa americana L.). O experimento foi conduzido em blocos casualizados, avaliando-se seis espécies fúngicas: Glomus clarum, Glomus etunicatum, Glomus manihots, Gigaspora albida, Acaulospora scrobiculata e Scutellospora heterogama, com dez repetições. As espécies A. scrobiculata, G. clarum e G. etunicatum colonizaram mais intensamente o sistema radicular e promoveram melhor desenvolvimento das mudas de jenipapeiro quando comparados a G. manihots e G. albida. O fungo G. etunicatum destacou-se, promovendo incrementos na altura $(44,4 \%)$; no diâmetro do caule $(63,6 \%)$; na produção de biomassa seca na parte aérea $(288,8 \%)$, nas raízes $(248,7 \%)$ e na área foliar (315,7\%) em comparação às mudas controle. Com exceção de $\mathrm{Mn}$ e Fe, mudas inoculadas apresentaram teores de nutrientes superior às mudas controle. As mudas que receberam inóculo de $S$. heterogama apresentaram crescimento e teor de nutrientes similares aos das mudas controle. A colonização micorrízica correlacionou-se positivamente com os teores de N, P, K, Mg e Cu e negativamente com os teores de Fe e Mn nas folhas das mudas de jenipapeiro. O jenipapeiro é uma planta responsiva aos FMA e a inoculação beneficiou o crescimento e a nutrição das mudas.
\end{abstract}

Palavras-chave - Jenipapo. Planta-nutrição. Fungos micorrízicos.

\begin{abstract}
Some studies have shown that inoculation with arbuscular mycorrhizal fungi (AMF) in seedling production has great potential for developing a rational and efficient cultivation of fruit tree seedlings. The objective of this study was to evaluate the effect of inoculation of arbuscular mycorrhizal fungi on growth and nutrition of seedlings of genipap (Genipa americana L.). The experiment was conducted in a randomized block design, evaluating six fungal species: Glomus clarum, Glomus etunicatum, Glomus manihots, Gigaspora albida, Acaulospora scrobiculata and Scutellospora heterogama with ten repetitions. The species A. scrobiculata, G. clarum and G. etunicatum colonized the root system more intensely and promoted better growth of genipap seedlings in comparison to G. manihots and G. albida. The fungus G. etunicatum stood out, promoting increases in height (44.4\%), stem diameter $(63.6 \%)$, dry biomass production in shoots $(288.8 \%)$, roots $(248.7 \%)$ and leaf area $(315.7 \%)$ compared to control seedlings. With the exception of $\mathrm{Mn}$ and $\mathrm{Fe}$, inoculated seedlings had higher nutrient content than the control seedlings. The seedlings inoculated with $S$. heterogama showed growth and nutrient content similar to the control seedlings. The mycorrhizal colonization correlated positively with $\mathrm{N}, \mathrm{P}, \mathrm{K}, \mathrm{Mg}$ and $\mathrm{Cu}$ content and negatively with $\mathrm{Fe}$ and $\mathrm{Mn}$ content in the leaves of genipap seedlings. Genipap is a plant that responds to AMF, and the inoculation favoured growth and nutrition of the seedlings.
\end{abstract}

Key words - Jenipapo. Plant nutrition. Mycorrhizal fungi.

\footnotetext{
*Autor para correspondência

${ }^{1}$ Recebido para publicação em 04/06/2010; aprovado em 27/07/2011

Tese; Pesquisa?

${ }^{2}$ Centro de Ciências Agrárias, Ambientais e Biológicas/UFRB, Rua Rui Barbosa, Campus Universitário, Cruz das Almas-BA, Brasil, 44.380-000, ferminosoares@gmail.com, cssagro@yahoo.com.br

${ }^{3}$ Universidade Federal do Vale do São Francisco/UNIVASF, Campus de Ciências Agrárias, Juazeiro-BA, Brasil, 48.902-300, garridoms@yahoo.com.br ${ }^{4}$ Centro de Ciências Agrárias, Ambientais e Biológicas/UFRB, Rua Rui Barbosa, Campus Universitário, Cruz das Almas-BA, Brasil, 44.380-000, fsousalima@yahoo.com.br
} 


\section{Introdução}

A produção brasileira de frutas aumentou 19\% entre 2001 e 2009, segundo dados do Instituto Brasileiro de Geografia e Estatística (IBGE). O Brasil possui condições climáticas favoráveis para o cultivo de fruteiras tropicais, o que tem colocado o país entre os principais produtores mundiais de frutos "in natura" e de sucos, despertando o interesse de produtores das diversas regiões onde os pomares são explorados comercialmente durante todo o ano e são responsáveis pelo abastecimento de todo o país (SILVEIRA; GOMES, 2007).

O jenipapo (Genipa americana L.), da família Rubiaceae, é uma espécie secundária tardia, com características de clímax, de crescimento moderado que ocorre em todo país. Essa espécie pode ser usada na arborização urbana e é também uma boa opção para os pequenos agricultores, tanto pela madeira como pelos frutos que são comestíveis e muito apreciados na fabricação de suco, doce, vinho e licor. Existe ainda o uso das cascas, raízes, folhas e sementes, na medicina popular (COSTA et al., 2005). Contudo, apesar do grande potencial alimentício, econômico e social da cultura, ainda perduram vários entraves de natureza agronômica, dentre eles a falta de mudas selecionadas (PRUDENTE, 2002).

As micorrizas arbusculares (MA) são associações entre raízes de plantas e fungos do solo do filo Glomeromycota (SCHÜßLER et al., 2001), conhecidos como fungos micorrízicos arbusculares (FMA). A ocorrência dos FMA é tão ampla que mais de $80 \%$ das plantas podem formar micorrizas arbuscular (JEFFRIES et al., 2003), sendo esta considerada uma associação cosmopolita, reconhecida como parte importante e integral dos ecossistemas naturais de todo o mundo (GADKAR et al., 2001). Os benefícios dessa simbiose, expressos principalmente como o estímulo ao crescimento vegetal, devem-se a fatores nutricionais, principalmente ao aumento da absorção de nitrogênio (COSTA; LOVATO, 2004), potássio e, especialmente, fósforo (CALVET et al., 2003). Os FMA, além de melhorar o estado nutricional, aumentam a tolerância a doenças radiculares (BORGES et al., 2007), e aceleram o crescimento e melhoram o vigor das mudas na sua fase de formação (LINDERMANN; DAVIES, 2001).

Alguns trabalhos têm demonstrado que a inoculação de fungos micorrízicos arbusculares (FMA) promove a formação de mudas de boa qualidade de diversas fruteiras (MARTINS et al., 2000; SOARES; MARTINS, 2000). O emprego de FMA pode otimizar a produção de mudas de mamoeiro (TRINDADE et al., 2001) e melhorar o crescimento e a nutrição das plantas de gravioleira (CHU et al., 2001). Diferenças no desenvolvimento em relação à micorrização também foram observadas em plantas de citros (SOUZA et al., 1997), maracujá (CAVALCANTE et al., 2002) e banana (DECLERCK et al., 2002).
A produção de mudas micorrizadas de espécies frutíferas deve atender a alguns requisitos como a seleção de FMA eficientes para o maior número possível de variedades da mesma espécie de planta, em determinada faixa de fertilidade do solo ou substrato, principalmente em relação ao nível de $\mathrm{P}$ disponível, além de ser competitivo em relação aos FMA nativos em condições de solo ou substrato não desinfestado (SILVEIRA; GOMES, 2007). Novas pesquisas devem ser desenvolvidas para selecionar espécies de FMA para produção de mudas micorrizadas de espécies frutíferas de interesse agronômico. Neste sentido, o presente trabalho teve como objetivo avaliar o efeito da inoculação com fungos micorrízicos arbusculares no crescimento e nutrição de mudas de jenipapeiro.

\section{Material e métodos}

O experimento foi conduzido com delineamento experimental em blocos casualizados com dez repetições em condições de casa-de-vegetação. Avaliou-se o efeito da inoculação com seis espécies de fungos micorrízicos: Glomus clarum Nicolson \& N.C.Schenck, Glomus etunicatum Becker \& Gerdemann, Glomus manihots Howelwe, Sieverding \& Schenck, Gigaspora albida Schenck \& Smith, Acaulospora scrobiculata Trappe $e$ Scutellospora heterogama (TH Nicolson \& Gerd.) C. Walker \& FE Sanders, no crescimento e nutrição de mudas de jenipapeiro. O tratamento testemunha constituiu-se de mudas de jenipapeiro não inoculadas.

O solo utilizado no experimento, foi coletado na profundidade de $0-20 \mathrm{~cm}$, seco ao ar, destorroado, homogeneizado, peneirado em peneira de $2 \mathrm{~mm}$. Os resultados da análise química do solo, determinada segundo EMBRAPA, (1999), foram: $\mathrm{pH}\left(\mathrm{H}_{2} \mathrm{O}\right)=6,4$; $\mathrm{Ca}=3,0 \mathrm{cmol}_{\mathrm{c}} \mathrm{dm}^{-3} ; \mathrm{Mg}=1,5 \mathrm{cmol}_{\mathrm{c}} \mathrm{dm}^{-3} ; \mathrm{Al}=0,05 \mathrm{cmol}_{\mathrm{c}}$ $\mathrm{dm}^{-3} ; \mathrm{P}=27 \mathrm{mg} \mathrm{dm}{ }^{-3} ; \mathrm{K}=61 \mathrm{cmol}_{\mathrm{c}} \mathrm{dm}^{-3} ; \mathrm{Na}=0,04 \mathrm{cmol}_{\mathrm{c}}$ $\mathrm{dm}^{-3} ; \mathrm{H}^{+}+\mathrm{Al}^{+3}=1,81 \mathrm{cmol}_{\mathrm{c}} \mathrm{dm}^{-3} ;$ M.O. $=16,08 \mathrm{~g} \mathrm{dm}^{-3}$.

Sementes de jenipapeiro foram desinfestadas com solução de hipoclorito de sódio $1 \%$ por dois minutos, seguido de três lavagens consecutivas em água destilada e semeadas em bandejas de plástico, contendo areia lavada e esterilizada. Após a germinação, as mudas foram transplantadas para sacos pretos de polietileno $(10 \times 25 \mathrm{~cm})$, contendo $400 \mathrm{~cm}^{3}$ de uma mistura de solo e areia, na proporção de $3: 1(\mathrm{v} / \mathrm{v})$, previamente esterilizados em autoclave a $120{ }^{\circ} \mathrm{C}$ durante uma hora e meia, em três dias alternados. No momento do transplantio, cada muda foi inoculada, colocando-se junto às raízes, com o auxílio de uma pipeta Pasteur, uma suspensão aquosa contendo 200 esporos da espécie fúngica. Dez $\mathrm{mL}$ de uma suspensão obtida do solo inóculo diluído em água e filtrado em peneira de $50 \mu \mathrm{m}$, portanto, isenta de propágulos de FMA, foram adicionados em todos os 
vasos, visando equilibrar a população microbiana do solo. As mudas foram mantidas em condições de casade-vegetação, sendo irrigadas diariamente com água destilada, durante 120 dias.

Após este período, fez-se a medição da altura das plantas (distância entre o nível do solo e a inserção do broto terminal da haste principal), com auxílio de uma régua milimetrada, e do diâmetro do caule a cinco centímetros acima do colo da planta utilizando-se um paquímetro digital. As mudas foram coletadas, separando-se a parte aérea das raízes. As raízes foram lavadas e, com auxílio de uma pinça, raízes finas $(<2 \mathrm{~mm})$ foram coletadas aleatoriamente e preservadas em etanol a $50 \%$. O restante das raízes foi colocado em estufa de secagem com ventilação forçada a $65^{\circ} \mathrm{C}$ até a obtenção de massa constante para determinação da biomassa seca radicular. A área foliar foi calculada pelo Programa SIARCS 3,0 e, em seguida, a parte aérea foi colocada para secar em estufa de secagem com ventilação forçada a $65^{\circ} \mathrm{C}$ até atingir massa constante. Após este período foi determinada a biomassa seca da parte aérea, e em seguida, foi realizada moagem e digestão nitroperclórica deste material para determinação dos teores de P, K, Ca, Mg, Fe, Cu, e Mn (EMBRAPA, 1999). O N foi determinado após digestão sulfúrica, pelo método de Kjeldahl (EMBRAPA, 1999).

Para determinar a porcentagem de colonização micorrízica, raízes finas $(<2 \mathrm{~mm})$ foram diafanizadas em $\mathrm{KOH} 10 \%$ a $90{ }^{\circ} \mathrm{C}$ em banho-maria durante 30 minutos, acidificadas em $\mathrm{HCl} 5 \%$ por 1 minuto, e em seguida coloridas com azul de metila $0,05 \%$ em lactoglicerol a $90{ }^{\circ} \mathrm{C}$ em banho-maria por 15 minutos (PHILLIPS; HAYMAN, 1970). Após coloridas, as raízes foram preservadas em frascos de vidro contendo glicerol ácido até a montagem das lâminas para microscopia. Foram montadas 10 lâminas por repetição, com 10 segmentos de $1 \mathrm{~cm}$ de comprimento de raiz, cobertas com glicerol ácido e lamínula. A contagem dos segmentos colonizados foi feita com auxílio de microscópio de luz (100x).
A eficiência micorrízica foi estimada conforme sugerido por Heidjen e Kuyper (2001), com base na produção de biomassa seca da parte aérea, calculada pela fórmula $E M=1-b / a$, onde $a=$ biomassa dos tratamentos de inoculação e $b=$ biomassa do tratamento não inoculado, sendo a simbiose benéfica para as plantas quando EM $>0$. Os resultados obtidos foram submetidos à análise de variância e teste de Scott $\&$ Knott $(\mathrm{p}<0,05)$ para a comparação das médias, utilizando o programa estatístico SISVAR 4.3. Os dados referentes à porcentagem de colonização micorrízica, foram transformados em arc sen $\sqrt{ } x / 100$. Foi calculado o coeficiente de correlação de Pearson usando percentual de colonização das raízes por fungos micorrízicos e os teores de nutrientes na parte aérea das plantas.

\section{Resultados e discussão}

Mudas de jenipapeiro inoculadas com as espécies fúngicas apresentaram incremento significativo na altura, variando de $25,3 \%$ a 44,4\%, com exceção daquelas colonizadas pelo fungo $S$. heterogama que não diferiram estatisticamente das mudas controle (TAB. 1). Influência positiva no diâmetro do caule foi observada nas mudas colonizadas por G. clarum $(58,1 \%)$, G. etunicatum $(63,6 \%)$ e $A$. scrobiculata (54,8\%), que não diferiram estatisticamente entre si. Os fungos G. manihots e G. albida não diferiram entre si, proporcionando incrementos de 41,8 e $49,7 \%$ respectivamente, no diâmetro do caule com relação ao controle. A colonização por S. heterogama proporcionou incremento de apenas 7,9\% no diâmetro do caule das mudas de jenipapeiro, não diferindo estatisticamente das mudas controle.

Os fungos $G$. etunicatum, $G$. clarum e $A$. scrobiculata se destacaram em aumentar a produção de massa seca da parte aérea e das raízes. Verificou-se um incremento de $288,8 \%$ na biomassa seca da parte aérea das mudas de jenipapeiro que receberam inóculo do

Tabela 1 - Crescimento de mudas de jenipapeiro inoculadas com fungos micorrízicos arbusculares

\begin{tabular}{lccccc}
\hline \multirow{2}{*}{ Espécie fúngica } & Altura das & Diâmetro do & Biomassa seca da & Biomassa seca das & \multirow{2}{*}{ Área foliar $\left(\mathrm{cm}^{2}\right)$} \\
\cline { 2 - 5 } & mudas $(\mathrm{cm})$ & caule $(\mathrm{mm})$ & parte aérea $\left(\mathrm{g} \mathrm{planta}^{-1}\right)$ & ${\text { raízes }\left(\mathrm{g} \mathrm{planta}^{-1}\right)}^{24,8 \mathrm{~d}}$ \\
\hline Controle & $4,03 \mathrm{~b}$ & $2,39 \mathrm{c}$ & $0,324 \mathrm{~d}$ & $0,197 \mathrm{~d}$ & $71,5 \mathrm{c}$ \\
G. manihots & $5,05 \mathrm{a}$ & $3,39 \mathrm{~b}$ & $0,873 \mathrm{c}$ & $0,411 \mathrm{c}$ & $89,9 \mathrm{~b}$ \\
G. clarum & $5,30 \mathrm{a}$ & $3,78 \mathrm{a}$ & $1,081 \mathrm{~b}$ & $0,588 \mathrm{~b}$ & $103,1 \mathrm{a}$ \\
G. etunicatum & $5,82 \mathrm{a}$ & $3,91 \mathrm{a}$ & $1,260 \mathrm{a}$ & $0,687 \mathrm{a}$ & $88,2 \mathrm{~b}$ \\
G. albida & $5,43 \mathrm{a}$ & $3,58 \mathrm{~b}$ & $0,811 \mathrm{c}$ & $0,505 \mathrm{c}$ & $79,7 \mathrm{c}$ \\
A. scrobiculata & $5,33 \mathrm{a}$ & $3,70 \mathrm{a}$ & $1,019 \mathrm{~b}$ & $0,478 \mathrm{c}$ & $27,3 \mathrm{~d}$ \\
S. heterogama & $4,39 \mathrm{~b}$ & $2,58 \mathrm{c}$ & $0,406 \mathrm{~d}$ & $0,205 \mathrm{~d}$ &
\end{tabular}

Médias seguidas da mesma letra não diferem estatisticamente pelo teste de Scott \& Knott $(\mathrm{p}<0,05)$ 
fungo G. etunicatum. Mudas colonizadas por G. clarum e A. scrobiculata também apresentaram incremento significativo de 233,6 e $214,5 \%$, respectivamente, na biomassa da parte aérea, em comparação às mudas controle. As mudas apresentaram menor incremento na biomassa seca da parte aérea quando colonizadas por $S$. heterogama $(25,3 \%)$, não diferindo estatisticamente das mudas controle. Com relação à biomassa seca das raízes, mudas colonizadas pelo fungo G. etunicatum apresentaram um incremento de $248,7 \%$ em relação às mudas controle. Observou-se também aumento significativo da produção de biomassa seca nas raízes das mudas que receberam inóculo do fungo G. clarum (198,5\%). Os resultados demonstraram que não houve diferença significativa no incremento promovido pelos fungos G. manihots (108,6\%), G. albida (156,3\%) e $A$. scrobiculata (142,6\%) na biomassa seca das raízes das mudas de jenipapeiro. Mudas inoculadas com o fungo $S$. heterogama não diferiram estatisticamente do observado nas mudas controle.

Os valores referentes à área foliar demonstraram que houve um incremento de $315,7 \%$ nas mudas colonizadas pelo fungo $G$. etunicatum. Incremento na área foliar das mudas de jenipapeiro foi também proporcionado pelos fungos $G$. clarum $(262,5 \%)$ e $G$. albida $(255,6 \%)$, com relação às mudas controle. Os menores valores de incremento na área foliar foram observados nas mudas colonizadas pelo fungo S. heterogama $(10,1 \%)$, não diferindo estatisticamente das mudas controle. Algumas espécies de FMA têm a capacidade de incrementar o tamanho das folhas de plantas frutíferas, favorecendo aumento da área fotossinteticamente ativa (SILVEIRA et al., 2002). Influência positiva da inoculação de FMA também foi observada na área foliar de mudas de maracujá amarelo (CAVALCANTE, 1999) e acerola (COSTA et al., 2001).

Parece ter ocorrido maior eficiência de algumas espécies de FMA em relação ao hospedeiro testado, tendo em vista que a resposta à inoculação resultou em crescimento diferenciado das mudas. Em geral, a inoculação de G. etunicatum destacou-se, promovendo maior incremento em todos os parâmetros de crescimento avaliados (TAB. 1), ao contrário do que aconteceu com as mudas inoculadas com $S$. heterogama, que em todas as características apresentaram valores similares aos das mudas controle. Apesar da ausência de especificidade, o estabelecimento da simbiose micorrízica é determinado por fatores edafoclimáticos e aspectos da relação fungoplanta. Assim, a utilização de diferentes espécies e/ou isolados de FMA resulta em respostas diferenciadas por parte do hospedeiro (CAVALCANTE et al. 2002; COSTA et al. 2001). Em outras situações, a associação com G. etunicatum não promoveu o crescimento de mangabeira (COSTA et al., 2001) e limoeiro-cravo (MELLONI et al., 2000) em relação ao controle, enquanto que somente porta-enxertos submetidos à inoculação de $S$. heterogama foram superiores à testemunha com relação ao acúmulo de matéria seca da parte aérea e raízes (SILVEIRA et al., 2002). Esses resultados demonstram, mais uma vez, a influência dos genótipos dos simbiontes na eficiência da simbiose, e a necessidade de estudos sobre a eficiência de espécies micorrízicas no desenvolvimento de mudas de diferentes espécies vegetais.

As mudas controle estavam sem colonização radicular e esporos na rizosfera. A porcentagem de colonização do sistema radicular variou de acordo com o fungo inoculado (TAB. 2). Mudas de jenipapeiro apresentaram maior colonização radicular quando associadas a $A$. scrobiculata (74,3\%), seguidas das colonizadas por G. etunicatum $(62,8 \%)$ e G. clarum (45,0\%). Contudo, quando avaliada a eficiência destes em beneficiar as mudas na produção de biomassa seca, observou-se que o fungo $G$. etunicatum $(74,0 \%)$ foi mais eficiente que $A$. scrobiculata $(68,0 \%)$ e $G$. clarum $(69,7 \%)$ que não diferiram estatisticamente entre si.

A eficiência da associação entre FMA e plantas é regulada pelos genótipos dos dois parceiros micorrízicos em interação com o meio ambiente, e não está necessariamente relacionada à porcentagem de colonização das raízes (COSTA et al., 2001). Em dado nível de colonização, a

Tabela 2 - Colonização e eficiência micorrízica dos fungos micorrízicos arbusculares em mudas de jenipapeiro

\begin{tabular}{lcc}
\hline \multicolumn{1}{c}{ Espécie fúngica } & Colonização radicular (\%) & Eficiência micorrízica (\%) \\
\hline G. manihots & $32,3 \mathrm{~d}$ & $61,6 \mathrm{c}$ \\
G. clarum & $45,0 \mathrm{c}$ & $69,7 \mathrm{~b}$ \\
G. etunicatum & $62,8 \mathrm{~b}$ & $74,0 \mathrm{a}$ \\
G. albida & $38,3 \mathrm{~d}$ & $56,7 \mathrm{c}$ \\
A. scrobiculata & $74,3 \mathrm{a}$ & $68,0 \mathrm{~b}$ \\
S. heterogama & $16,5 \mathrm{e}$ & $20,5 \mathrm{~d}$ \\
\hline
\end{tabular}

Médias seguidas da mesma letra não diferem estatisticamente pelo teste de Scott \& $\operatorname{Knott}(\mathrm{p}<0,05)$ 
eficiência da absorção de nutrientes pode ser afetada pelos parâmetros da troca de nutrientes na interface fungo-raiz e pela extensão, viabilidade e capacidade de transporte das hifas externas (MARSCHNER; DELL, 1994).

Apesarde apresentarem maiorgrau de colonização radicular, mudas que receberam inóculo do fungo $G$. albida (38,3\%) não diferiram estatisticamente das inoculadas por $G$. manihots $(32,3 \%)$. Comportamento similar foi observado quando avaliada a eficiência destes fungos em beneficiar as mudas de jenipapeiro na produção de biomassa seca. A eficiência da maioria das espécies de FMA avaliadas neste trabalho foi muito expressiva. $\mathrm{O}$ fato de as mudas de jenipapeiro sem inoculação terem se desenvolvido pouco, sugere possivelmente alta dependência micorrízica desta planta.

Mudas inoculadas com $S$. heterogama apresentaram baixo grau de colonização radicular (16,5\%), sendo este abaixo do nível mínimo (50\%) da colonização considerada efetiva (CARNEIRO et al., 1998). A menor eficiência simbiótica deste fungo na produção de biomassa seca das mudas $(20,5 \%)$ pode ser atribuída tanto a fatores genéticos, como também à intereção dos fatores que envolvem o ambiente, o fungo simbionte $S$. heterogama e a planta hospedeira jenipapeiro (SILVEIRA et al., 2002). Segundo Moreira e Siqueira (2006), G. clarum e G. etunicatum têm preferência por solos neutros ou pouco ácidos; enquanto que $A$. scrobiculata é indiferente ao $\mathrm{pH}$. O $\mathrm{pH}$ do solo a 6,4 pode ter beneficiado as espécies $G$. clarum, G. etunicatum e A. scrobiculata em detrimento da espécie $S$. heterogama.

Observou-se maiores teores de $\mathrm{N}$ nas mudas inoculadas com $G$. etunicatum e A. scrobiculata do que nas mudas inoculadas com as demais espécies às quais não diferiram estatisticamente das mudas controle (TAB. 3). As mudas submetidas à inoculação com A. scrobiculata apresentaram maior teor de $\mathrm{P}$ em relação às mudas com inoculação das demais espécies de FMA. Mudas inoculadas com as espécies de FMA tiveram maior teor de $\mathrm{K}$ em comparação com as mudas não inoculadas, com exceção das inoculadas com $S$. heterogama. G. manihots aumentou significativamente os teores de $\mathrm{Ca}$ e $\mathrm{Mg}$ nas mudas de jenipapeiro em relação às demais espécies fúngicas que não diferiram estatisticamente entre si. As mudas inoculadas com $S$. heterogama tiveram menores teores de $\mathrm{P}, \mathrm{K}, \mathrm{Ca}$ e $\mathrm{Mg}$, em relação às mudas inoculadas com as demais espécies de FMA, não diferindo estatisticamente das mudas controle.

Com relação aos micronutrientes, observou-se que o teor de $\mathrm{Cu}$ foi superior nas mudas inoculadas com as espécies fúngicas em relação às mudas controle. Durante alguns anos, a importância da micorriza no processo de absorção de micronutrientes pela planta era pouco enfatizada. Atualmente, a eficiência dos fungos micorrízicos em absorver $\mathrm{Cu}$ é constatada com freqüência (CHU et al., 2001; SENA et al., 2002, SILVA JÚNIOR et al., 2010; TRINDADE et al., 2001). Não houve diferença significativa entre as mudas inoculadas e não inoculadas com relação ao teor foliar de Mn. O teor de Fe foi significativamente maior nas mudas controle em comparação com as mudas inoculadas com as espécies fúngicas.

O efeito da micorrização no crescimento das plantas é predominantemente nutricional, sendo esse efeito promovido pela ramificação das hifas e pelo micélio externo que aumenta o volume de solo explorado para as regiões onde as raízes não alcançam, aumentando assim a absorção dos nutrientes, principalmente o fósforo

Tabela 3 - Teor foliar de nutrientes das mudas de jenipapeiro inoculadas com fungos micorrízicos arbusculares

\begin{tabular}{|c|c|c|c|c|c|c|c|c|}
\hline \multirow{2}{*}{ Espécie fúngica } & $\mathrm{N}$ & $\mathrm{P}$ & $\mathrm{K}$ & $\mathrm{Ca}$ & $\mathrm{Mg}$ & $\mathrm{Cu}$ & $\mathrm{Mn}$ & $\mathrm{Fe}$ \\
\hline & \multicolumn{5}{|c|}{$\left(\mathrm{g} \mathrm{kg}^{-1}\right)$} & \multicolumn{3}{|c|}{$\left(\mathrm{mg} \mathrm{kg}^{-1}\right)$} \\
\hline Controle & $8,42 \mathrm{~b}$ & $0,201 \mathrm{c}$ & $7,00 \mathrm{~b}$ & $3,98 \mathrm{c}$ & $4,38 \mathrm{c}$ & $9,96 \mathrm{~b}$ & $55,0 \mathrm{a}$ & $388,4 \mathrm{a}$ \\
\hline G. manihots & $10,12 b$ & $0,993 \mathrm{~b}$ & $10,40 \mathrm{a}$ & $5,68 \mathrm{a}$ & $6,52 \mathrm{a}$ & $13,90 \mathrm{a}$ & $44,6 \mathrm{a}$ & $203,0 \mathrm{~b}$ \\
\hline G. clarum & $9,54 \mathrm{~b}$ & $0,772 \mathrm{~b}$ & $9,80 \mathrm{a}$ & $4,92 \mathrm{~b}$ & $5,94 \mathrm{~b}$ & $12,16 \mathrm{a}$ & $40,0 \mathrm{a}$ & $216,0 \mathrm{~b}$ \\
\hline G. etunicatum & $14,18 \mathrm{a}$ & $0,840 \mathrm{~b}$ & $9,00 \mathrm{a}$ & $4,84 \mathrm{~b}$ & $5,90 \mathrm{~b}$ & $13,70 \mathrm{a}$ & $42,0 \mathrm{a}$ & $163,0 \mathrm{~b}$ \\
\hline G. albida & $10,66 \mathrm{~b}$ & $0,648 \mathrm{~b}$ & $9,40 \mathrm{a}$ & $4,80 \mathrm{~b}$ & $5,42 \mathrm{~b}$ & $12,94 \mathrm{a}$ & $45,8 \mathrm{a}$ & $179,0 \mathrm{~b}$ \\
\hline A. scrobiculata & $13,50 \mathrm{a}$ & $1,404 \mathrm{a}$ & $9,20 \mathrm{a}$ & $4,86 \mathrm{~b}$ & $5,86 \mathrm{~b}$ & $12,90 \mathrm{a}$ & $39,6 \mathrm{a}$ & $146,8 \mathrm{~b}$ \\
\hline S. heterogama & $9,38 \mathrm{~b}$ & $0,224 \mathrm{c}$ & $7,80 \mathrm{~b}$ & $4,06 \mathrm{c}$ & $4,70 \mathrm{c}$ & $12,14 \mathrm{a}$ & $48,0 \mathrm{a}$ & $235,4 \mathrm{~b}$ \\
\hline
\end{tabular}

Médias seguidas da mesma letra na vertical não diferem entre si pelo teste de Scott \& Knott $(\mathrm{p}<0,05)$ 
(MOREIRA; SIQUEIRA, 2006). A inoculação de mudas de mamoeiro com fungos micorrízicos proporcionou aumento na absorção de fósforo, potássio e cobre (TRINDADE et al., 2001) e dos conteúdos de fósforo e potássio na parte aérea de mudas micropropagadas de bananeira (SAMARÃO et al., 2000). Silva Júnior et al., (2010) verificaram que mudas de meloeiro inoculadas com fungos micorrízicos apresentavam maiores teores de Fe $(102,1 \%), \mathrm{Cu}(153,2 \%), \mathrm{Zn}(82,6 \%)$ e Mn $(92,4 \%)$ em relação às mudas controle sem inoculação.

O coeficiente de correlação indica o grau de relacionamento entre as características da planta e do FMA, sendo completamente independentes as características que não apresentam correlação (BENTIVENGA et al., 1997). Os dados indicam sete correlações significativas, do total de oito entre a colonização radicular e os teores de nutrientes nas folhas das mudas de jenipapeiro (TAB. 4). Correlações significativas entre os nutrientes nas folhas e as percentagens de colonização por fungos micorrízicos nas raízes são indicativos de alguma contribuição das micorrizas na absorção desses nutrientes (OLIVEIRA; OLIVEIRA, 2005).

Tabela 4 - Coeficiente de correlação linear simples entre a colonização micorrízica (\%) e o teor foliar de nutrientes das mudas de jenipapeiro

\begin{tabular}{cl}
\hline Nutriente & Valor r \\
\hline $\mathrm{Cu}$ & $0,522^{* *}$ \\
$\mathrm{Fe}$ & $-0,684^{* * *}$ \\
$\mathrm{Mn}$ & $-0,504^{* *}$ \\
$\mathrm{Ca}$ & $0,423^{\mathrm{NS}}$ \\
$\mathrm{Mg}$ & $0,585^{* * *}$ \\
$\mathrm{~K}$ & $0,434 * *$ \\
$\mathrm{P}$ & $0,795 * * *$ \\
$\mathrm{~N}$ & $0,714 * * *$
\end{tabular}

Ns: não-significativo $(\mathrm{p}>0,05) ; * *(\mathrm{p}<0,01), * * *(\mathrm{p}<0,001)$

Não foi observado correlação entre a colonização das raízes pelos FMA e o teor de Ca nas folhas. Observou-se uma correlação negativa entre as colonizações por FMA e os teores de Fe e Mn.
Houve correlações positivas variando entre baixa e média entre a colonização radicular e os teores de $\mathrm{Cu}$, $\mathrm{Mg}$ e $\mathrm{K}$. Os baixos valores de $\mathrm{r}$ indicam que outros fatores não relacionados com a colonização dos fungos micorrízicos nas raízes estão atuando mais intensamente na eficiência da absorção dos nutrientes. Correlação superior a 0,707 foi observada apenas para $\mathrm{P}$ e $\mathrm{N}$, confirmando o esperado, visto que o estabelecimento da associação micorrízica geralmente esta relacionado com a absorção de $\mathrm{P}$ e $\mathrm{N}$ do solo (MARSCHNER; DELL, 1994).

Resultados obtidos neste estudo servirão como suporte para otimizar o processo de produção de mudas de jenipapeiro, uma vez que, a associação micorrízica proporcionou melhoria no crescimento vegetativo e nutrição das mudas. O estudo demonstrou também que geralmente existe seletividade do hospedeiro por determinadas espécies de FMA, e esta seletividade não é para eficiência e sim, para sobrevivência.

\section{Conclusões}

1.O jenipapeiro é uma planta responsiva a associação micorrízica;

2. As espécies Glomus etunicatum, Acaulospora scrobiculata e Glomus clarum são mais promissoras como inóculo, enquanto que Scutellospora heterogama não é eficiente em promover o crescimento e absorção de nutrientes pelas mudas de jenipapeiro;

3.A associação micorrízica favorece a absorção de nutrientes como $\mathrm{N}, \mathrm{P}, \mathrm{K}, \mathrm{Ca}, \mathrm{Mg}$ e $\mathrm{Cu}$ pelas mudas de jenipapeiro.

\section{Referências}

BENTIVENGA, S. P.; BEVER, J. D.; MORTON, J. B. Genetic variation of morfological characters within a single isolate of the endomycorrhizal fungus Glomus clarum (Glomaceae). American Journal of Botany, v. 84, n. 09, p. 1211-1216, 1997.

BORGES, A. J. S. et al. Reduction of fusarium wilt of "bananamaçã" by inoculation of arbuscular mycorrhizal fungi. Pesquisa Agropecuária Brasileira, v. 42, n. 01, p. 35-41, 2007.

CALVET, C. et al. Aptitude for mycorrhizal root colonization in Prunus rootstocks. Scientia Horticulturae, v. 09, n. 01, p. 1-10, 2003.

CARNEIRO, M. A. C. et al. Micorriza arbuscular em espécies arbóreas e arbustivas nativas de ocorrência no sudeste do Brasil. Cerne, v. 04, n. 01, p. 129-145, 1998. 
CAVAlCANTE, U. M. T. Efeitos da associação de fungos micorrízicos arbusculares com o maracujazeiro-marelo Passiflora edulis Sims. F. flavicarpa Deg.). 1999. 132 f. Tese (Doutorado em Biologia de Fungos). Recife, Universidade Federal de Pernambuco.

CAVAlCANTE, U. M. T. et al. Influência da densidade de esporos de fungos micorrízicos arbusculares na produção de mudas de maracujazeiro amarelo. Pesquisa Agropecuária Brasileira, v. 37, n. 05, p. 643-649, 2002.

CHU, E. Y.; MÖLLER, M. R. F.; CARVALHO, J. G. Efeito da inoculação micorrízica em mudas de gravioleira em solo fumigado e não fumigado. Pesquisa Agropecuária Brasileira, v. 36. n. 04, p. 671-680, 2001

COSTA, C. M. C. et al. Influência de fungos micorrízicos arbusculares sobre o crescimento de dois genótipos de aceroleira (Malpighia emarginata D.C.). Pesquisa Agropecuária Brasileira, v. 36, n. 06, p. 893-901, 2001.

COSTA, M. D.; LOVATO, P. E. Fosfatases na dinâmica do fósforo do solo sob culturas de cobertura com espécies micorrízicas e não-micorrízicas. Pesquisa Agropecuária Brasileira, v. 39, n. 06, p. 603-605, 2004.

COSTA, M. C. et al. Substratos para produção de mudas de jenipapo (Genipa americana L.). Pesquisa Agropecuária Tropical, v. 35, n. 01, p. 19-24, 2005.

DECLERCK, S.; RISEDE, J-M.; DELVAUX, B. Greenhouse response of micropropagated bananas inoculated with in vitro monoxenically produced arbuscular mycorrhizal fungi. Scientia Horticulturae, v. 93,n. 03/04, p. 301-309, 2002.

EMPRESABRASILEIRADEPESQUISAAGROPECUÁRIA (EMBRAPA). Manual de análises químicas de solos, plantas e fertilizantes. In: SILVA, F. C. (Ed.) Brasília: Embrapa Comunicação para Transferência de Tecnologia de Tecnologia, 1999. $370 \mathrm{p}$.

JEFFRIES, P. et al. The contribution of arbuscular fungi in sustainable maintenance of plant health and soil fertility. Biology and Fertility of Soils, v. 37, p. 1-16., 2003.

GADKAR, V.; DAVID-SCHWARTZ, R.; KUNIK, T.; KAPULNIK, Y. Arbuscular mycorrhizal fungal colonization. Factors involved in host recognition. Plant Physiology, v. 127, n. 04, p. 1493-1499, 2001.

HEIJDEN, E. W.; KUYPER, T. W. Does origin of mycorrhizal fungus or mycorrhizal plant influence effectiveness of the mycorrhizal symbiosis? Plant and Soil, v. 230, n. 02, p. 161-174, 2001.

LIDERMANN, R. G.; DAVIES, A. Comparative response of selected grapevine rootstocks and cultivars to inoculation with different mycorrhizal fungi. American Journal Ecology Viticulture, v. 52, n. 01, p. 1-9, 2001.

MARSCHNER, H.; DELL, B. Nutrient uptake in mycorrhizal symbiosis. Plant and Soil, v. 159, n. 01, p. 89-102, 1994.

MARTINS, M. A.; GONÇALVES, G. F.; SOARES, A. C. F. Efeito de fungos micorrízicos arbusculares associados a compostos fenólicos, no crescimento de mudas de mamoeiro. Pesquisa Agropecuária Brasileira, v. 35, n. 07, p. 1465-1471, 2000.

MELLONI, R. et al. Fósforo adicionado e fungos micorrízicos arbusculares no crescimento e nutrição mineral de limoeiro-cravo [Citrus limonia (L) Osbeck]. Revista Brasileira de Ciência do Solo, v. 24, p. 767-775, 2000.

MOREIRA, F. M. S.; SIQUEIRA, J. O. Microbiologia e bioquímica do solo. 2. ed. Lavras: Editora UFLA, 2006. 626 p.

OLIVEIRA, N. A.; OLIVEIRA, L. A. Colonização por fungos micorrízicos arbusculares e teores de nutrientes em cinco cultivares de bananeiras em um latossolo da amazônia. Revista Brasileira de Ciência do Solo, v. 29, p. 481-488, 2005.

PHILLIPS, J. M.; HAYMAN, D. S. Improved procedures for clearing root and staining parasitic and vesicular-arbuscular mycorrhizal fungi for rapid assessment of infection. Transaction British Mycological Society, v. 55, n. 01, p. 158-161, 1970.

PRUDENTE, R. M. Jenipapo (Genipa americana L). In: NETO, R. D. V. (Ed). Fruteiras potenciais para os tabuleiros costeiros e baixadas litorâneas. Aracajú: EMBRAPA-TABULEIROS COSTEIROS/Empresa de Desenvolvimento Agropecuário de Sergipe - EMDAGRO, 2002. p. 89-114.

SAMARÃO, S. S.; MARTINS, M. A.; TEIXEIRA, L. S. Produção de mudas micropropagadas de bananeira (Musa sp.,) inoculadas com fungos micorrízicos arbusculares. Revista Brasileira de Fruticultura, v. 22, n. 02, p. 20002004, 2000.

SCHÜßLER A.; SCHWARZOTT D.; WALKER C. A new fungal phylum, the Glomeromycota: phylogeny and evolution. Mycological Research, v. 105, p. 1413-1421, 2001.

SILVA JÚNIOR, J. M. T. et al. Desenvolvimento de meloeiro associado a fungos micorrízicos arbusculares e cultivado em substrato pó de coco. Revista Brasileira de Ciências Agrárias, v. 05, n. 01, p. 54-59, 2010.

SILVEIRA, S. V.; SOUZA, P. V. D.; KOLLER, O. C. Efeito de fungos micorrízicos arbusculares no desenvolvimento do abacateiro. Pesquisa Agropecuária Brasileira, v. 37, n. 11, p. 1597-1604, 2002.

SILVEIRA, A. P. D.; GOMES, V. F. F. Micorrizas arbusculares em plantas frutíferas tropicais. In: SILVEIRA, A. P. D.; FREITAS. S. S. (Ed.) Microbiota do solo e qualidade ambiental. Campinas: Instituto Agronômico, 2007. p. 57-78.

SENA, J. O. A.; LABATE, C. A.; CARDoso, E. J. B. N. Micronutrient accumulation in mycorrhizal citrus under different phosphorus regimes. Acta Scientiarum, v. 24, n. 05, p. 1265-1268, 2002.

SOARES, A. C. F.; MARTINS, M. A. Influência de fungos micorrízicos arbusculares, associada à adição de compostos fenólicos, no crescimento de mudas de maracujazeiro-amarelo (Passiflora edulisf.flavicarpus). Revista Brasileira de Ciências do Solo, v. 24, n. 04, p. 731-740, 2000. 
SOUZA, P. V. D. et al. Desenvolvimento do citrange 'Troyer' infectado com fungo micorrízico, em dois substratos de cultivo. Pesquisa Agropecuária Brasileira, v. 32, n. 10, p. 1039-1045, 1997.
TRINDADE, A. V. et al. Estimativa do coeficiente de determinação genotípica em mamoeiros (Carica papaya L.) inoculados com fungo micorrízico arbuscular. Revista Brasileira de Fruticultura, v. 23, n. 03, p. 607-612, 2001. 